\title{
LA EDUCACIÓN AMBIENTAL HACIA EL DESARROLLO SUSTENTABLE
}

ENVIRONMENTAL EDUCATION TOWARDS SUSTAINABLE DEVELOPMENT

Gloria Castillo Osorio*

*Doctora en Derecho. Profesora Investigadora de la Universidad Juárez Autónoma de Tabasco. ORCID: https://orcid.org/0000-0002-2717-5666.

Dirección para correspondencia: castillosorio@hotmail.com

Fecha de recibido: 4 de junio de 2019

DOI: https://doi.org/10.19136/hitos.a25n73.3534

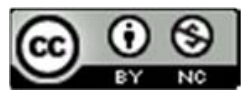

CASTILLO-OSORIO G. 
LA EDUCACIÓN AMBIENTAL HACIA EL DESARROLLO SUSTENTABLE.

\section{RESUMEN}

El propósito del presente trabajo es ofrecer una perspectiva de la contribución de la Educación Ambiental como parte de un proceso que permite aminorar los desequilibrios y daños que se causan al medio ambiente, con miras hacia el desarrollo sustentable; considera la aplicación de medidas técnicas, políticas económicas y socio-educativas. En este trabajo, se utilizará el término sustentable, tal como lo marcan los ordenamientos jurídicos.

OBJETIVO: Analizar la contribución de la Educación Ambiental en su desarrollo conceptual en función de alcanzar el desarrollo sustentable, mediante la mejora, protección y conservación del ambiente como fuente de sustento de las sociedades presentes y futuras.

MATERIAL Y MÉTODO: Es una investigación de tipo documental y emplea los métodos descriptivo y analítico, se revisó la literatura legislativa y especializada, ideas doctrinarias, ordenamientos jurídicos internacionales y nacionales, así como revistas científicas, que brindaron un marco teórico y jurídico del significado de la Educación Ambiental hacia un desarrollo sustentable.

RESULTADOS: Se determina la contribución de la Educación Ambiental en los diversos niveles de la educación formal, no formal e informal, para lograr una concienciación de la sociedad, que a través de conocimientos, valores, actitudes y aptitudes permita evaluar el significado de la naturaleza como base de la sobrevivencia.

CONCLUSIONES: La Educación Ambiental, como proceso, conduce a determinar que los problemas ambientales presentan características, tales como: globalizador, complejo, transdisciplinario, integral, continúo e interdisciplinario; conlleva a la participación activa en la solución de problemas, su importancia en el desarrollo sustentable y estimula la conciencia de los retos que se deben enfrentar. La educación forma parte de la cultura, del respeto a los derechos humanos, sobre todo el derecho a un medio ambiente sano, vinculado a los aspectos ecológicos, económicos, sociales, políticos, y éticos, que posibilita y considera pertinente promover la capacitación de los formadores de recursos humanos en las cuestiones ambientales.

PALABRAS CLAVES: Educación. Medio ambiente. Desarrollo sustentable. 
LA EDUCACIÓN AMBIENTAL HACIA EL DESARROLLO SUSTENTABLE.

\section{ABSTRACT}

The purpose of the present work is to offer a perspective of the contribution of Environmental Education as part of a process that allows to reduce the imbalances and damages that are caused to the environment, with a view towards sustainable development; it also considers the application of technical measures, economic and socio-educational policies.

OBJECTIVE: To analyze the contribution of Environmental Education in its conceptual development in order to achieve sustainable development, through the improvement, protection and conservation of the environment as a source of livelihood for present and future societies.

MATERIAL AND METHOD: It is a documentary research and uses descriptive and analytical methods; legislative and specialized literature, doctrinal ideas, national and international legal systems as well as scientific journals were reviewed, which provided a theoretical and legal framework of the meaning of the Environmental Education towards sustainable development.

RESULTS: The contribution of Environmental Education is determined at the various levels of formal, non-formal and informal education, to raise awareness of society, which through knowledge, values, attitudes and skills can be able to assess the meaning of nature as the basis of survival.

CONCLUSIONS: Environmental Education as a process, leads to determine that environmental problems show characteristics such as: globalizing, complex, transdisciplinary, comprehensive, continuous and interdisciplinary; It leads to active participation in problem solving; its importance in sustainable development stimulates awareness of the challenges that must be faced. Education that is part of: the culture, the respect for human rights, especially the right to a healthy environment, which is linked to ecological, economic, social, political, and ethical aspects; and considers relevant and makes it possible to promote the training of educators in environmental issues.

KEYWORDS: Education. Environment. Sustainable development. 
LA EDUCACIÓN AMBIENTAL HACIA EL DESARROLLO SUSTENTABLE.

\section{INTRODUCCIÓN}

La naturaleza es anterior a la aparición del hombre en este planeta, este binomio hombrenaturaleza, aparece cuando el primero depende para su subsistencia de los recursos que brinda la propia naturaleza; sin embargo, se ha presentado una disposición desmedida que lleva a plantear un desequilibrio del ecosistema, por ende se debe tomar conciencia de que todos los seres vivos dependen del medio ambiente, que la naturaleza la brinda en calidad de préstamo.

Al ir en aumento los problemas ambientales, se justifica prolongar la atención del tema de la Educación Ambiental, principalmente para ser parte de los contenidos curriculares de la enseñanza-aprendizaje que permita la formación integral de los estudiantes, y el logro de una convivencia social; capacitar a todos en el compromiso consigo mismo, con la sociedad y con el entorno. El reto es hacer conciencia del significado del medio ambiente en el desarrollo humano.

Se trata de un proceso, que debe ser continuo y permanente, donde la enseñanza formal, no formal e informal examine los principales aspectos del entorno desde un punto de vista local hacia una perspectiva internacional de modo que los educandos se compenetren y se concienticen sobre el tema. La pregunta es, ¿cómo se logra el desarrollo sustentable?

\section{Problemas ambientales}

Uno de los desafíos más importantes que enfrenta la sociedad, tanto en el ámbito nacional como en el internacional, es encontrar una forma equilibrada de conciliar dos realidades y tomar conciencia; por un lado, satisfacer las necesidades y por el otro, preservar el medio ambiente.

Los problemas ambientales deben ser estudiados de forma integral, ya que al ser un sistema se conforma por partes que se encuentran relacionadas entre sí, los componentes están integrados en el medio físico, biótico, económico y sociocultural (García-Reategui, 2007). Existen una serie de problemas, a continuación se mencionan unos cuantos: deterioro del suelo; desertificación; deforestación; contaminación del agua, aire, suelo, subsuelo; cambio climático; daño a la capa de ozono; manejo de residuos sólidos y peligrosos; pérdida de biodiversidad; incremento poblacional; el menoscabo de saberes tradicionales; pobreza; 


\section{LA EDUCACIÓN AMBIENTAL HACIA EL DESARROLLO SUSTENTABLE.}

seguridad alimentaria; soberanía alimentaria; y la fractura hidráulica; problemas y materias que son fundamentales estudiarlas desde la mirada de la Educación Ambiental.

Luchar contra la degradación ambiental depende de la participación de todos los sectores de la sociedad, de la educación y sensibilidad de la población, del respeto por los valores éticos y espirituales y por la diversidad cultural, así como de la protección de los conocimientos indígenas (Carmona, 2015). La aportación del derecho económico es fundamental para encontrar las soluciones apropiadas, dar respuesta a las múltiples preguntas y brindar fundamentos jurídicos a los problemas ambientales, con el espectro amenazante del cambio climático, el aumento de la escasez de agua, la capacidad de expandir el consumo de la creciente clase media mundial, la creciente pobreza y la amenaza de la hambruna global.

Es preocupante el deterioro de los recursos naturales, lo que afecta la vida de los seres humanos, donde la atención de la comunidad científica internacional se enfoca en el fomento de la concienciación para utilizar responsablemente el saber de todos los campos de la ciencia y darle respuesta a estos problemas que impactan en el desarrollo y sobrevivencia de todos en este planeta, vinculados con un sistema de desarrollo económico y social. Constantemente se modifica la naturaleza, incrementando el desequilibrio ambiental, es así que surge el concepto de Educación Ambiental, en aras de crear conciencia acerca de este problema, conocer sus causas, sus consecuencias y tratar de darle solución.

El modelo de la enseñanza-aprendizaje que en la actualidad permea es por competencia, fomentar la conciencia en los educandos de la problemática ambiental; que le permita obtener los conocimientos hacia un desarrollo sustentable mediante la promoción de actitudes, aptitudes, valores y conocimientos, en beneficio de la sustentabilidad; educación que debe ser permanente y adecuada a las circunstancias en que se hoy se vive. Conformada por valores ambientales que den una relación de compromiso con el medio ambiente, de importancia crucial, ya que guarda estrecha relación con los saberes ambientales.

Los métodos de la interdisciplinariedad y los principios de la complejidad orientan el conocimiento ambiental (Leff, 2003), es una nueva cultura en la que se construyen nuevas visiones y se despliegan nuevas estrategias de producción sustentable y democracia participativa y se reafirma que la racionalidad ambiental debe ejercerse en todos los ámbitos sociales, que se funda en el saber ambiental que tiene como principio a la complejidad. 


\section{LA EDUCACIÓN AMBIENTAL HACIA EL DESARROLLO SUSTENTABLE.}

Incumbe preparar a las presentes y futuras generaciones, para ser reflexivos y juiciosos ante los desequilibrios y desastres naturales; y para estar en posibilidad de resolverlos. Mediante la formación de recursos humanos en valores, en conceptos, que desarrolle las habilidades y actitudes para una convivencia armónica con su cultura y su medio ambiente. Se debe asumir que una de las funciones de la Educación Ambiental es ser conscientes de lo compleja que es la naturaleza, por ello con apoyo de estrategias pedagógicas se generará una conciencia crítica y responsable para un estilo de vida en armonía con la naturaleza. Camarena (2006) sostiene que el: hábitat humano no es simplemente un mundo de objetos, sino un mundo de valores.

El papel de la escuela ha de consistir en la reconstrucción, reelaboración y en la ampliación de estas estructuras, con un enfoque globalizador, que, por medio de la perspectiva interdisciplinar, permita utilizar sus concepciones para la solución de problemas de su realidad (Martínez, 2015).

Esta educación sistémica, posee un papel sustancial al fomentar los valores y modificar la conducta de la sociedad de manera positiva, en relación con los bienes naturales. Se debe reconocer que el avance de la ciencia y la tecnología ha sido benéfico; pero también ha sido la base de un deterioro y desequilibrio ambiental, por lo tanto es pertinente introducir la Educación Ambiental que posibilite, ya sea de forma individual o colectiva, evitar o disminuir el impacto negativo y lograr un desarrollo sustentable que permita que generaciones presentes y futuras, disfruten y gocen de forma racional de lo que la naturaleza ofrece para su progreso.

Urge que la educación ambiental este inmersa en todos los programas de estudio de todos los niveles, que abone en el orgullo de los seres humanos de vivir en este planeta, confiar en un presente, donde el derecho humano a un medio ambiente sea sano, se pueda disfrutar y se brinde de igual manera a los futuros pobladores del planeta.

Los niveles de participación donde el aprendizaje ambiental puede incidir son:

- Educación formal: Enseñanza preescolar, primaria, secundaria y superior, así como el personal docente y los profesionales en formación.

- El sector de la educación no formal: Jóvenes y adultos (individual o colectivo) de todos los segmentos de la población: familias, trabajadores, directivos y ejecutivos, asociaciones culturales o deportivas. 


\section{LA EDUCACIÓN AMBIENTAL HACIA EL DESARROLLO SUSTENTABLE.}

- El sector de la educación informal: Es provocada por la interacción con el ambiente como la vida familiar, el trabajo los medios de comunicación.

Martínez (2017) señala, que el aprendizaje significativo o de calidad, se caracteriza por implicar que el educando comprenda conceptos, procedimientos, actitudes y valores, será significativo si el aprendizaje se relaciona con lo que el alumno ya sabe y si éste adopta la actitud de aprendizaje correspondiente para hacerlo así. La enseñanza debe lograr que el estudiante sea crítico, que se interese en la práctica con aportaciones positivas para contribuir a la resolución del problema y valore los resultados obtenidos.

La finalidad, es formar ciudadanos responsables, para mejorar la calidad de vida mediante la apropiación de valores ambientales y de la convivencia democrática. Orientar hacia la resolución de los problemas medio ambientales, hacia la toma de decisiones y hacia la acción.

\section{Aportaciones de organismos internacionales}

Un punto interesante es que el concepto de desarrollo sustentable o sostenible está aún en construcción, el término sostenible ha perdido significado, ciertamente tienen aristas comunes, pero lo sustentable hace relación a la armonía que existe entre lo económico, lo social, lo ambiental con el sistema de valores. En cambio, lo sostenible considera a cada uno de dichos subsistemas por separado (Zara, 2018).

Priorizar uno de los tres ejes (económico, social y ambiental) es afectar a los otros dos, por ejemplo, si la humanidad desaparece, con ella todo lo demás, en cambio la naturaleza con el paso del tiempo se podrá equilibrar. La historia de este proceso educativo, se origina a partir de la reflexión que se realiza en la segunda mitad del siglo $X X$, con relación a los modelos económicos con base en la idea de que los recursos naturales eran infinitos; esta preocupación por la crisis ambiental es motivo para diversas reuniones internacionales. Una de las evidencias es la publicación del libro: La Primavera Silenciosa ${ }^{1}$ de Rachel Carson, donde hace visible la contaminación causada por la Revolución Verde, el uso de pesticidas químicos, fungicidas y herbicidas que dieron como origen un impacto negativo en los recursos naturales.

\footnotetext{
1 “Los futuros historiadores quizás no comprendan nuestro desviado sentido de la proporción. ¿Cómo pueden los seres inteligentes tratar de dominar unas cuantas especies molestas por un método que contamine todo lo que les rodea y les atraiga la amenaza de un mal e incluso de la muerte de su propia especie? $\mathrm{Y}$, sin embargo, esto es precisamente lo que hemos hecho. Lo hemos hecho, no obstante, por razones que se derrumban en cuanto las examinamos." http://www.scielo.org.mx/pdf/remcf/v3n14/v3n14a1.pdf
}

CASTILLO-OSORIO G.

SEPTIEMBRE-DICIEMBRE 2019. Año 25, Número 73. Págs. 355-370 


\section{LA EDUCACIÓN AMBIENTAL HACIA EL DESARROLLO SUSTENTABLE.}

Es importante llamar la atención, al referente avance de los acuerdos internacionales que se iniciaron por parte de la Organización de las Naciones Unidas para la Educación, la Ciencia y la Cultura (UNESCO), tal como la Declaración de Estocolmo (1972), donde por vez primera y de manera definitiva aparecen los términos ambiente y desarrollo, (principios 1 y 2), dimensión ambiental considerada como una condición y límite del crecimiento económico y uso de los recursos naturales; se crea el Programa de las Naciones Unidas sobre el Medio Ambiente, enfocado a fomentar la conciencia ecológica y la cooperación entre los sectores sociales y las entidades para trabajar en favor de la conservación del medio ambiente.

En ese mismo año se publicó el Primer Informe del Club de Roma conocido como: Los límites del crecimiento económico, (Tetreault, 2008), donde se analizan los cinco componentes que determinan y limitan el crecimiento en el mundo: 1) la población; 2) la producción agrícola; 3) los recursos no renovables; 4) la producción industrial, y 5) la contaminación; con la gran preocupación de que el planeta colapsaría hacia el año 2000, si no se tomaban acciones inmediatas.

Es de resaltar, que la Conferencia de las Naciones Unidas sobre el Medio Humano (1972) en su Recomendación 96 dirigida específicamente entre otras instituciones a la UNESCO, solicita la adopción de las disposiciones necesarias a fin de establecer un programa internacional de educación sobre el medio, interdisciplinario y con carácter escolar y extraescolar, que abarque todos los niveles de enseñanza y se dirija al público en general, con miras a enseñarle las medidas sencillas que, dentro de sus posibilidades pueda tomar para ordenar y controlar su medio. El principio 19, alude a la Educación Ambiental, dirigida a jóvenes y adultos, que estén bien informados de cómo debe ser una conducta responsable en la protección y mejoramiento del medio ambiente.

Han pasado más de cuatro décadas desde que se plasmó esta Recomendación y aún se continúa insistiendo en ser reflexivos y conscientes de la responsabilidad que se debe asumir en relación al cuidado del medio ambiente que le da sustento material al hombre para su desarrollo desde diversos enfoques (intelectual, moral, social y espiritualmente).

En la Carta de Belgrado del Seminario Internacional de Educación Ambiental (1975) se plasman los objetivos del proceso educativo, a saber: a) Conciencia, b) Conocimientos, c) Actitudes, d) Aptitudes, e) Capacidad de evaluación y finalmente f) Participación; aseverando la necesidad 


\section{LA EDUCACIÓN AMBIENTAL HACIA EL DESARROLLO SUSTENTABLE.}

de una ética individual y reafirmando que el destinatario principal es el público en general. Otro acontecimiento trascendental es la Conferencia Intergubernamental de Educación Ambiental de Tbilisi (1977), en la cual se presentan objetivos, principios rectores, finalidades y estrategias, así como el concepto de Educación Ambiental. El documento señala, la necesidad de una ética más humana, donde se adopten actitudes y comportamientos, que reconozca y responda de manera sensible a las relaciones complejas, y en constante cambio entre el hombre y la naturaleza y entre los mismos hombres.

La Organización de las Naciones Unidas (ONU), probó la Declaración sobre el Derecho al Desarrollo (1986) y una parte fundamental del cumplimiento de este derecho se vincula necesariamente al principio de justicia y equidad ambiental; se confirma que el: "derecho al desarrollo es un derecho humano inalienable y que la igualdad de oportunidades para el desarrollo es una prerrogativa tanto de las naciones como de los individuos que componen las naciones".

La Comisión Mundial del Medio Ambiente (1987), presenta "Nuestro Futuro Común" (Informe Brundland), donde: "plantea inicialmente el concepto de desarrollo sostenible que orientará la estrategia educativa ambiental; además de las ideas que sometió a revisión el concepto de desarrollo económico y la emergencia de la crítica ambientalista al modo de vida contemporáneo". Desarrollo sostenible, se refiere a aquel: "que satisface las necesidades presentes sin comprometer la habilidad de las generaciones futuras para satisfacer sus propias necesidades" (Carmona, 192).

Diez años posteriores al Congreso Intergubernamental, se realizó el Congreso Internacional de Educación y Capacitación Ambiental (1987), que elaboró la Estrategia Internacional de Acción en Materia de Educación y Formación Ambientales para el Decenio de 1990, donde se reconoce que el origen de los problemas ambientales son los factores sociales, económicos y culturales, enfocando la actuación de los valores, las actitudes y los comportamientos individuales y colectivos. Analiza los principios y las características esenciales de la educación y de la formación ambiental.

Las consideraciones anteriores, se amplían en el Programa 21, que nace de la Cumbre de la Tierra o como se conoce también Conferencia de Naciones Unidas sobre el Medio Ambiente y el Desarrollo (1992), el objetivo principal fue introducir un programa extenso y un plan nuevo 


\section{LA EDUCACIÓN AMBIENTAL HACIA EL DESARROLLO SUSTENTABLE.}

para la acción internacional, sus recomendaciones iban desde nuevos métodos educativos, hasta formas de preservar los recursos naturales, pasando por nuevos caminos para participar en el diseño de una economía sostenible.

En Guadalajara, México (1992), se realizó el primer Congreso Iberoamericano de Educación Ambiental, como inicio de muchos otros que posteriormente se realizaron. Su principal motivo fue sentar las bases de una estrategia para el futuro. En ese mismo año, se llevó a cabo el Foro Global o Foro de la Gente, en Río de Janeiro, donde se aprobó el Tratado de Educación Ambiental para Sociedades Sostenibles y de Responsabilidad Global; el punto 16 alude que la Educación Ambiental debe ayudar a desarrollar una conciencia ética sobre todas las formas de vida, respetar los ciclos vitales de la naturaleza e imponer límites a la explotación de esas formas de vida.

En Sajonia, Grecia, se llevó a cabo la Conferencia Internacional Medio Ambiente y Sociedad: Educación y Sensibilización para la Sostenibilidad, (1997) se pretende reorientar la educación en el sentido de la sostenibilidad como objetivo conceptual primordial, obtener soluciones ante los problemas ambientales que necesitan ser solventados para alcanzar condiciones mínimas de sobrevivencia sobre la superficie terrestre.

En la Conferencia sobre Medio Ambiente y Desarrollo Humano en la Cumbre de la Tierra Rio+20 celebrada en el 2012 en Rio de Janeiro, se reconoció el poco avance que en materia de sostenibilidad se ha logrado y se enfatiza la importancia del concepto desarrollo sostenible, y el deseado para el bienestar humano hoy y mañana, y para crear conciencia entre los docentes para cambiar su forma de pensar y sentir, para educar en la sostenibilidad, ya que la educación es el medio más efectivo que la sociedad posee para enfrentar los retos del futuro.

Las Naciones Unidas declaran que del 2005 al 2015, sería la Década de la Educación para el Desarrollo Sostenible, su finalidad era satisfacer las necesidades del mundo actual sin comprometer la capacidad de las futuras generaciones para enfrentar las necesidades que se les presenten; proporcionar la oportunidad de adquirir competencias, conocimientos y valores, que permitan contribuir a un futuro humano justo, económicamente viable y ecológicamente sostenible. Se define a la Educación Ambiental como un: "proceso que dura toda la vida, cuyo objetivo... es crear conciencia ambiental, conocimiento ecológico, actitudes, valores, 


\section{LA EDUCACIÓN AMBIENTAL HACIA EL DESARROLLO SUSTENTABLE.}

compromiso para las acciones y responsabilidades éticas para el uso racional de los recursos con el propósito de lograr un desarrollo adecuado y sustentable".

Desde 1972 se han realizado infinidad de conferencias, congresos, foros, que han hecho suyos los contenidos que marcan diversos instrumentos internacionales en materia de Educación Ambiental y Desarrollo Sustentable. Se comparte la importancia del tema a lo largo de la historia y en este sentido abunda e insiste Novo (2003), en que la sostenibilidad no es solo cuestión de economistas, sino, también de filósofos, ecólogos, artistas, antropólogos,... de todos aquellos que, en definitiva, pueden ayudar a comprender los diferentes valores tangibles e intangibles de la vida sobre la tierra.

Goodland et al (1992), comparte el contenido de cinco dimensiones de la sustentabilidad: a) dimensión económica, debe modificar el principio de "el que contamina paga" por el de "lo que paga es prevenir la contaminación". El discurso en relación con este principio ha sido cuestionado, en virtud de que se sostiene que se paga por contaminar, cuando su origen es otro; b) dimensión humana, el desarrollo sustentable se orienta a una mejor calidad de vida, reasignando los recursos económicos para atender estas necesidades, avanzar hacia la formación de capital humano y social, c) Dimensión ambiental, no es posible concebir el desarrollo ni la vida humana sin el sustento de la naturaleza, d) Dimensión institucional, la sustentabilidad implica realizar progresos significativos en la descentralización política administrativa de las decisiones, para estimular nuevas formas de organización y participación ciudadana, e) Dimensión tecnológica, implica la búsqueda y cambio hacia tecnologías más eficientes, en el caso de los países industrializados deben tener tecnologías eficientes, limpias y apropiadas.

Martínez (2015) señala, la teoría de las inteligencias múltiples que permite a los docentes transformar las actuales clases y unidades didácticas en actividades de aprendizaje multimodal para los alumnos. Lo que se busca como facilitadores del aprendizaje es que cada alumno se desarrolle y tenga las herramientas para saber lo que aprende y para qué lo aprende. En este contexto la Educación Ambiental, centrada en valores éticos y conocimientos, necesita de la reflexión y el compromiso como mecanismos que permitan lograr sociedades sustentables que dispongan de los recursos de forma racional y responsable. 


\section{LA EDUCACIÓN AMBIENTAL HACIA EL DESARROLLO SUSTENTABLE.}

En México, la evolución de este proceso educativo, se contempla en diversos ordenamientos jurídicos, se indican a continuación los más relevantes. La Constitución Política de los Estados Unidos Mexicanos en su numeral 25, dispone que al Estado le corresponde la rectoría del desarrollo nacional, garantizando que sea integral y sustentable.

En la Ley General del Equilibrio Ecológico y Protección Ambiental se desprende en el artículo 3, fracción $\mathrm{XI}$, la definición de Desarrollo Sustentable como: "el proceso evaluable mediante criterios e indicadores del carácter ambiental, económico y social que tiende a mejorar la calidad de vida y la productividad de las personas, que se funda en medidas apropiadas de preservación del equilibrio ecológico, protección del ambiente y aprovechamiento de recursos naturales, de manera que no se comprometa la satisfacción de las necesidades de las generaciones futuras". La fracción XXXVIII puntualiza el concepto de Educación Ambiental como: proceso de formación dirigido a toda la sociedad, tanto en el ámbito escolar como en el ámbito extraescolar, para facilitar la percepción integrada del ambiente a fin de lograr conductas más racionales a favor del desarrollo social y del ambiente... comprende la asimilación de conocimientos, la formación de valores, el desarrollo de competencias y conductas con el propósito de garantizar la preservación de la vida. El numeral 158, menciona dentro de las funciones de la Secretaría de Medio Ambiente y Recursos Naturales impulsar el fortalecimiento de la conciencia ecológica, a través de la realización de acciones conjuntas con la comunidad para la preservación y mejoramiento del ambiente, el aprovechamiento racional de los recursos naturales y el correcto manejo de desechos.

La Ley General de Educación, en su artículo 7, fracción XI tiene entre sus fines: XI.- Inculcar los conceptos y principios fundamentales de la ciencia ambiental, el desarrollo sustentable, la prevención del cambio climático, así como de la valoración de la protección y conservación del medio ambiente como elementos esenciales para el desenvolvimiento armónico e integral del individuo y la sociedad.

La visión normativa es vasta (Ley General de Cambio Climático, Ley de Desarrollo Rural Sustentable, Ley General de Desarrollo Forestal Sustentable, Ley General para la Prevención y Gestión Integral de los Residuos, Ley de Aguas Nacionales, por citas algunas); sus disposiciones están referidas a la creación de programas, campañas de educación, capacitación, formación profesional, investigación científica, actualización de contenidos curriculares, cuyo punto central es el desarrollo sustentable. Leyes enfocadas a lograr un nuevo 


\section{LA EDUCACIÓN AMBIENTAL HACIA EL DESARROLLO SUSTENTABLE.}

sistema de valores, conocer el medio ambiente, fomentar la conciencia, el respeto y cuidado de los recursos naturales; sin embargo, aún queda mucho por hacer.

Como parte de la Educación Ambiental, se necesita estar informado; por ejemplo, en el caso de los estudiosos del derecho, conocer que se trata de un tema que merece darle la atención debida, en aras de una aplicación correcta de la ley, conocerla e interpretar correctamente su contenido y su alcance, para que sea obedecida. Entonces, la educación servirá de medio para lograr estas metas. Las instituciones educativas, deben orientar su actuar en la demanda de nuevos conocimientos y actitudes del docente, donde los alumnos sean críticos y propositivos.

Molano (2014) considera que lo más adecuado en el contexto de las instituciones de educación superior es hablar de formación ambiental. Esta formación de recursos humanos se enfocará en el cuidado y conservación del medio ambiente, por los problemas ambientales que se están viviendo, para que conjuntamente se enfrenten estos retos, ya que cada día está en peligro la sobrevivencia.

La experiencia docente, permite identificar la falta de sensibilidad para considerar como prioritarios los conocimientos, valores, aptitudes y actitudes hacia el logro del desarrollo sustentable $\mathrm{y}$, por ende hacia el planeta que es la casa donde se habita; sin embargo, la habilidad y capacidad del docente consiste en lograr el interés del educando de conocer cada vez más sobre la temática, de estimular la conciencia y la responsabilidad de actos en favor del medio ambiente, reflexionar sobre los daños ambientales, no únicamente en el presente sino hacia el futuro. La enseñanza no consiste en impartir la materia, sino que se trata de un proceso más complejo para que el conocimiento se aprehenda y el estudiante conozca cómo lograr la solución de problemáticas ambientales.

\section{CONCLUSIONES}

La Educación Ambiental, como proceso, conduce a determinar que los problemas ambientales presentan características, tales como: globalizador, complejo, transdisciplinario, integral, continúo e interdisciplinario; conlleva a la participación activa en la solución de problemas, su importancia en el desarrollo sustentable, y estimula la conciencia de los retos que se deben enfrentar. 


\section{LA EDUCACIÓN AMBIENTAL HACIA EL DESARROLLO SUSTENTABLE.}

La Educación Ambiental, debe formar parte de la cultura, del respeto a los derechos humanos, sobre todo el derecho a un medio ambiente sano, vinculado a los aspectos ecológicos, económicos, sociales, políticos, y éticos, que posibilite y considere pertinente promover la capacitación de los formadores de recursos humanos en las cuestiones ambientales, así como forjar una conciencia desde el plano universitario, familiarizar los conceptos, métodos y medios para el desarrollo.

Actualmente, aún no existe consenso y objetivos claros para alcanzar un desarrollo que tenga la característica de sustentable, donde la educación juega un papel importante. Se han emitido diversos discursos en diferentes tiempos y ámbitos como el gubernamental, el científico, el académico, el social, donde cada uno realiza aportaciones desde su contexto sobre el desarrollo sustentable.

\section{REFERENCIAS BIBLIOGRÁFICAS}

Camarena, B. (2006). La educación ambiental en el marco de los foros internacionales: una alternativa de desarrollo. Estudios Sociales, 15(28), 7-42.

Carmona, M. (2015). Reflexiones en torno al derecho económico y su vínculo con el derecho ambiental: hacia el paradigma de la sustentabilidad. México: Instituto de Investigaciones Jurídicas de la UNAM. Recuperado de https://archivos.juridicas.unam.mx/www/bjv/libros/8/3984/13.pdf

Conferencia de las Naciones Unidas sobre el Medio Ambiente Humano. (1972). Declaración de Estocolmo sobre el medio ambiente humano. Recuperado de http://www.ordenjuridico.gob.mx/TratInt/Derechos\%20Humanos/INST\%2005.pdf

Congreso Internacional UNESCO-PNUMA sobre la Educación y la Formación Ambientales. (1987). Elementos para una estrategia internacional de acción en materia de educación y formación ambientales para el decenio de 1990. Recuperado de https://unesdoc.unesco.org/ark:/48223/pf0000075072_spa/PDF/075072spab.pdf.multi

Constitución Política de los Estados Unidos Mexicanos (2018, 27 de agosto). Diario oficial de la federación. Febrero $\quad$ 5, $19917 . \quad$ Recuperado de http://www.diputados.gob.mx/LeyesBiblio/pdf/1_090819.pdf 
LA EDUCACIÓN AMBIENTAL HACIA EL DESARROLLO SUSTENTABLE.

Declaración de la Conferencia de las Naciones Unidas sobre el Medio Humano. Recuperado de https://www.dipublico.org/conferencias/mediohumano/A-CONF.48-14-REV.1.pdf

Foro Global. (1992). Tratado de educación ambiental hacia sociedades sostenibles $y$ responsabilidad global. Río de Janeiro. Recuperado de https://sites.google.com/site/historiaeducacionambiental/decada-de-los-

90/1992?tmpl=\%2Fsystem\%2Fapp\%2Ftemplates\%2Fprint\%2F\&showPrintDialog=1

García, J. y Reategui, R. (2007). La educación ambiental en la sociedad globalizada. Didáctica de las Ciencias Experimentales y Sociales, (21), 151-158. Recuperado de https://dialnet.unirioja.es/servlet/articulo?codigo=2476022

Goodland, R. et al. (1992). Medio ambiente y desarrollo sostenible. Más allá del Informe Brundtland. Madrid: Trotta.

Leff, E. (2003). La complejidad ambiental. México: Siglo Veintiuno Editores.

Ley General de Cambio Climático. (2012). Diario Oficial de la Federación. Recuperado de http://www.diputados.gob.mx/LeyesBiblio/pdf/LGCC_130718.pdf

Ley General de Educación. (1993). Diario oficial de la federación. Recuperado de https://www.sep.gob.mx/work/models/sep1/Resource/558c2c24-0b12-4676-ad908ab78086b184/ley_general_educacion.pdf

Ley General del Equilibrio Ecológico y la Protección al Ambiente. (1988). Diario oficial de la federación. Recuperado de http://www.diputados.gob.mx/LeyesBiblio/pdf/148_050618.pdf

Martínez, M. (2015). Incorporación de la educación ambiental como eje transversal a través del diseño de unidades didácticas. Trabajo presentado en el XI Congreso Nacional de Investigación Educativa, México, D.F. Recuperado de http://www.comie.org.mx/congreso/memoriaelectronica/v11/docs/area_03/1802.pdf

Martínez, R. (2017). Ensayo crítico sobre educación ambiental. Revista Electrónica Diálogos Educativos, 12(24), 74-104. Recuperado de http://revistas.umce.cl/index.php/dialogoseducativos/article/view/1056

Molano, A. y Herrera, J. (2014). La Formación Ambiental en la Educación Superior: Una Revisión Necesaria. Revista Luna Azul, (39). Recuperado de http://www.scielo.org.co/pdf/luaz/n39/n39a12.pdf

CASTILLO-OSORIO G. 
LA EDUCACIÓN AMBIENTAL HACIA EL DESARROLLO SUSTENTABLE.

Naciones Unidas. (1992). Conferencia de las naciones unidas sobre el medio ambiente y el desarrollo. Recuperado de https://www.un.org/spanish/conferences/wssd/unced.html

Novo, M. (2003). El desarrollo sostenible: sus implicaciones en los procesos de cambio. Polis: Revista Latinoamericana, (5). Recuperado de https://journals.openedition.org/polis/6908

Seminario Internacional de Educación Ambiental. (1975). La carta de Belgrado, un marco general para la educación ambiental. Recuperado de https://unesdoc.unesco.org/ark:/48223/pf0000017772_spa/PDF/017772spab.pdf.multi

Tetreault, D. (2008). En torno al medio ambiente: una revisión de cuatro debates. Espiral, 14(42), 41-72. Recuperado

de http://www.scielo.org.mx/scielo.php?script=sci_arttext\&pid=S1665$05652008000300002 \&$ Ing=es\&tlng=es.

Zabala, I. y García, M. (2008). Historia de la educación ambiental desde su discusión y análisis en los congresos internacionales. Revista de Investigación, 32(63). Recuperado de https://dialnet.unirioja.es/servlet/articulo?codigo=2547197

Zarta, P. (2018). La sustentabilidad o sostenibilidad: un concepto poderoso para la humanidad. Tabula Rasa, (28), 409-423. DOI 10.25058/20112742.n28.18 